\title{
Ad-hoc Networks Energy Consumption: A review of the Ad-Hoc Routing Protocols
}

\author{
V. Kanakaris*, D. Ndzi and D. Azzi \\ Department of Electronic and Computer Engineering, University of Portsmouth, United Kingdom.
}

Received 6 December 2009; Revised 9 June 2010; Accepted 6 July 2010

\begin{abstract}
In MANETs (Mobile Ad Hoc Networks) communication at the mobile nodes can be achieved by using multihop wireless links. The architecture of such network is based not on a centralized base station but on each node which acts as a router and forwards data packets to other nodes in the network. The aim of each protocol, in an ad-hoc network, is to find valid routes between two communicating nodes. These protocols must be able to handle high mobility of the nodes which often cause changes in the network topology. This paper evaluates four ad-hoc network protocols (AODV, DSDV, DSR and TORA) in different network scales taking into consideration the mobility factor. The evaluation of these four protocols was carried out using Network Simulator-2 (ns2), and the poor performance of TORA may be attributed to its implementation in this package. Therefore further investigation of TORA implementation in ns 2 needs to be carried out.
\end{abstract}

Keywords: Ad-hoc Network, Sensor Network, Power Aware Routing, Routing Protocols, AODV, DSDV, DSR, TORA.

\section{Introduction}

\section{Wireless Networks}

Wireless systems, both mobile and fixed, have become an indispensable part of communication infrastructure. Their applications range from simple wireless low data rate transmitting sensors to high data rate real-time systems such as those used for monitoring large retail outlets or real-time broadcasting of sport events. The existing wireless technology is based on point-to-point technology. An example is GSM system with an architecture that is based on mobile nodes communicating directly with central access points. Sometimes there are networks which cannot rely on the centralized connectivity such as Mobile Ad-Hoc Networks (MANET). MANET is a wireless network having mobile nodes with no fixed infrastructure. These kinds of networks are used in areas such as environmental monitoring or in rescue operations. The main limitation of ad-hoc systems is the availability of power. In addition to running the onboard electronics, power consumption is governed by the number of processes and overheads required to maintain connectivity.

A number of protocols have been developed for non-centralised networks, e.g. Temporally Order Routing Algorithm (TORA) [1] TORA is a protocol for multi-hop networks. The choice of a route in a multi-hop network influences the performance of the network, measured in terms of power consumption. There are some protocols that strive for energy efficient routing such as DSR (Dynamic Source Routing [2], AODV (Ad-Hoc On Demand Routing) [3] and DSDV (Destination-Sequenced Distance Vector) [4]. These protocols offer varying degrees of efficiency.
This research focuses on communication protocols specifically aimed at limiting power consumption and prolonging battery life whilst maintaining the robustness of the system. It also proposes further research into more efficient protocols or variants of existing protocols such as TORA [1] and network topologies. Emphasis is on protocols that could be suitable for the implementation of scalable systems in high node density environments such as in manufacturing or product distribution industries. The main objective of this paper is to analyze the TORA protocol for efficiency in terms of power and suggest ways it could be improved. This will be made by measuring the energy with respect to different network size and taking into consideration the remaining battery power.

\section{Types of MANET Routing Protocols}

The MANET routing protocols [5] are mainly developed to maintain route inside MANET, and they do not use any access points to connect to other nodes in the network and Internet. Routing protocols can be classified into three categories depending on their properties. The classifications are:

$>$ Centralized versus Distributed

$>$ Static versus Adaptive

$>$ Reactive versus Proactive

In centralized algorithms, all route choices are made by a central node, while in distributed algorithms, the computation of routes is shared among the network nodes. In static algorithms, the 
route used by source destination pairs is fixed regardless of traffic condition. It can only change in response to a node or link failure. This type of algorithm cannot achieve high throughput under a broad variety of traffic input patterns. In adaptive routing, the routes used to route between source-destination pairs may change in response to congestion. A third classification that is more related to ad-hoc networks is to classify the routing algorithms as either proactive or reactive.

\section{Proactive (Table-Driven) Routing Protocols}

In this family of protocols, nodes maintain one or more routing tables about nodes in the network. These routing protocols update the routing table information either periodically or in response to change in the network topology. The advantage of these protocols is that a source node does not need route-discovery procedures to find a route to a destination node. On the other hand the drawback of these protocols is that maintaining a consistent and up-to-date routing table requires substantial messaging overhead, which consumes bandwidth and power, and decreases throughput, especially in the case of a large number of high node mobility. There are various types of Table Driven Protocols: Destination Sequenced Distance Vector routing (DSDV), Wireless routing protocol (WRP) [6], Fish eye State Routing protocol (FSR), Optimized Link State Routing protocol (OLSR), Cluster Gateway Switch Routing protocol (CGSR), Topology Dissemination Based on Reverse Path Forwarding (TBRPF).

\section{Reactive (On-Demand) Routing Protocols}

For protocols in this category there is an initialisation of a route discovery mechanism by the source node to find the route to the destination node when the source node has data packets to send. When a route is found, the route maintenance is initiated to maintain this route until it is no longer required or the destination is not reachable. The advantage of these protocols is that overhead messaging is reduced. One of the drawbacks of these protocols is the delay in discovering a new route. The different types of reactive routing protocols are: Dynamic Source Routing (DSR) [6], Ad-hoc On-Demand Distance Vector routing (AODV) and Temporally Ordered Routing Algorithm (TORA).

This paper is organized as follows. In Section II, a description of the TORA, DSDV, DSR and AODV routing protocols are presented. In Section III, the ns2 software and the metrics that are taken into consideration in the simulation are given whilst the results are presented in Section IV. Section V concludes the paper and proposes ideas for future work.

\section{Description of selected Routing Protocols}

TORA: TORA [1] routing protocol is based on "link reversal" algorithm. Every node has information regarding it adjacent nodes. In this way TORA provides multiple routes for any pair of nodes. Moreover it has the ability to quickly follow the topological changes that may occurred and re-create valid routes. Hence when a node seeks a route to a given destination it sends a QUERY message which includes the address of the destination node. This packet travels through the network until it reaches the destination or an intermediate node that has a route to the destination node. The re- ceiver node then broadcasts an UPDATE packet listing the number of direct links that has been used in order to reach the destination. As this node propagates this UPDATE information packet through the network, each node updates its list by adding another pair of nodes (source-destination). This creates a series of directed links from the node that originated the QUERY to the destination node. When the node finds a specific destination which is unreachable, it sets a local maximum value of direct links for that destination. If the node cannot find any adjacent node which has a list of direct links to the destination, it attempts to search a new route. If there is a network partition then the node sends a CLEAR message that resets all routing states and removes invalid routes from the network. TORA operates on top of Internet MANET Encapsulation Protocol (IMEP) providing reliable delivery of route-messages and informs the routing protocol of any changes to the links to its neighbours. IMEP tries to aggregate IMEP and TORA messages into a single packet (called block) in order to reduce overhead. For link-status sensing and list maintenance, IMEP sends out periodic BEACON messages which are answered by each node that hears it with a HELLO reply message.

DSDV: In DSDV [4] protocol messages are exchanged between nearby mobile nodes (i.e. mobile nodes that are within range of one another). Routing updates may be triggered or routine. Updates are caused when routing information from one of the neighbours forces a change in the routing table. If there is a packet which the route to its destination is unknown it is cached while routing queries are sent out. The packets are cached until routereplies are received from the destination. The buffer has a size and time limit for caching packets beyond which packets are dropped. All packets which have destination to the mobile node are routed directly by the address dmux (dmux port hands the packets to the respective destination agents) to its port dmux. In the event that a target is not found (which happens when the destination of the packet is not the mobile node itself), the packets are forwarded to the default target which is the routing agent. The routing agent designates the next hop for the packet and sends it down to the link layer.

DSR: In DSR [2] protocol the agent checks every data packet for source-route information. The packets are then forwarded as per the routing information. In case it cannot find any routing information in the packet, it provides the source route if route is known and when the destination is not known it caches the packet and sends out route queries. The routing query is initially sent to all nearby nodes and is always triggered by a data packet which has no route information regarding its destination. Route-replies are sent back either through the destination node or by intermediate nodes if routing information to the destination is found.

AODV: AODV protocol [3] is a mixture of both DSR and DSDV protocols. It keeps the basic route-discovery and route-maintenance of DSR and uses the hop-by-hop routing sequence numbers and beacons of DSDV. When a node needs to know a route to a specific destination it creates a ROUTE REQUEST. Next the route request is forwarded by intermediate nodes which also create a reverse route for itself from the destination. When the request reaches a node with route to destination it creates again a ROUTE 
REPLY which contains the number of hops that are require to reach the destination. All nodes that participate in forwarding this reply to the source node create a forward route to destination. This route created from each node from source to destination is a hopby-hop state and not the entire route as in source routing.

\section{Simulation and Metrics}

The aim of these simulations is to analyze the TORA protocol by comparing it with other protocols (AODV, DSR, and DSDV) for its efficiency in terms of power as well as throughput. This has been made by measuring the energy with respect to different network size and taking into consideration the remaining battery power. The simulation tool that has been used in this study is ns2 [7]. Communication Management Unit's (CMU's) wireless extension to ns2 provides the implementation of the DSR, AODV, DSDV, TORA routing protocols. So ns2 is selected for evaluating these protocols.

\section{Parameters of the Simulation}

\begin{tabular}{|c|c|}
\hline Channel type & WirelessChannel \\
\hline Radio-propagation model & TwoRayGround \\
\hline Antenna type & OmniAntenna \\
\hline Interface queue type & DropTail/PriQueue \\
\hline Maximum packet in Queue & 50 \\
\hline Network interface type & Phy/WirelessPhy \\
\hline MAC type & 802_11 \\
\hline Topographical Area & $500 \times 300$ sq.m \\
\hline txPower & $0.5 W$ \\
\hline rxPower & $0.1 \mathrm{~W}$ \\
\hline idlePower & $0.01 \mathrm{~W}$ \\
\hline Initial energy of a Node & 1000.0 Joules \\
\hline Routing protocols & AODV/DSDV/DSR/TORA \\
\hline Number of mobile nodes & 10,20 and 30 \\
\hline Number of mobile nodes & $10,20,30,40,50,60,70,80,90,100$ \\
\hline Mobility & 0 or $20 \mathrm{~m} / \mathrm{s}$ \\
\hline
\end{tabular}

The performances of the four routing protocols with network size of 10, 20 and 30 nodes with mobility (mobility $20 \mathrm{~m} / \mathrm{s}$ ) have been assessed. In all the cases, only two senders Constant Bit-rate (CBR) over User Datagram Protocol (UDP) and two receivers (null sink) have been used.

\section{Metrics considered for Evaluation [8] Number of Packets dropped:}

This is the number of data packets that are not successfully sent to the destination during the transmission. In this study the time versus number of packets dropped have been calculated.

\section{Remaining Battery Power:}

The number of nodes in the network versus the average remaining battery power is considered as the metric to analyze the performance of the protocols in terms of power

\section{Consumed Power:}

The number of nodes in the network versus average consumed battery power is considered as a metric.

\section{Throughput:}

The throughput metric measures how well the network can constantly provide data to the sink. Throughput is the number of packet arriving at the sink per ms.

\section{MAC Load:}

This is the ratio of the number of MAC layer messages propagated by every node in the network to the number of data packets successfully delivered to all destination nodes. In other words, the MAC load is the average number of MAC messages generated to each data packet successfully delivered to the destination.

\section{Dropped Packets:}

This is the number of nodes in the network versus agent level total dropped packet.

\section{Dropped Bytes:}

This is the number of nodes in the network versus agent level total dropped bytes.

\section{Results and Analysis}

\section{The Simulation Results}

The following two graphs show the results of power analysis.

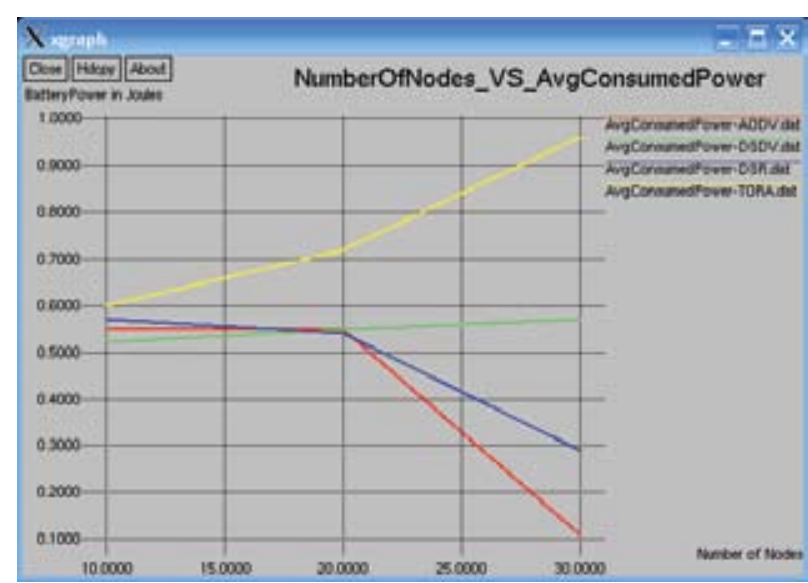

Figure 1. The average consumed power versus the number of nodes using 30 nodes.

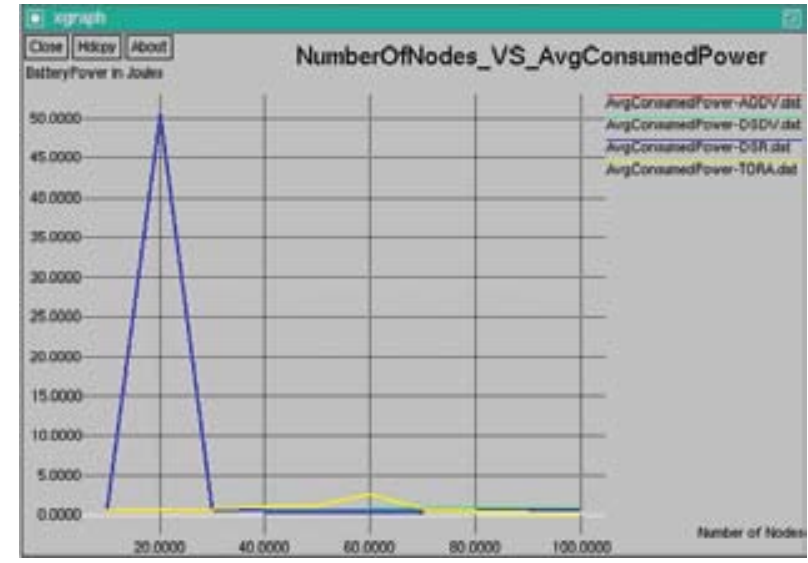

Figure 2. The average consumed power versus the number of nodes using 100 nodes. 
Figure 1 shows that the consumed power of networks using AODV and DSR decreases significantly when the number of nodes exceeds 20. On the contrary, the consumed power of a network using the TORA protocol increases rapidly whilst that of DSDV based network shows stability with increasing number of nodes.

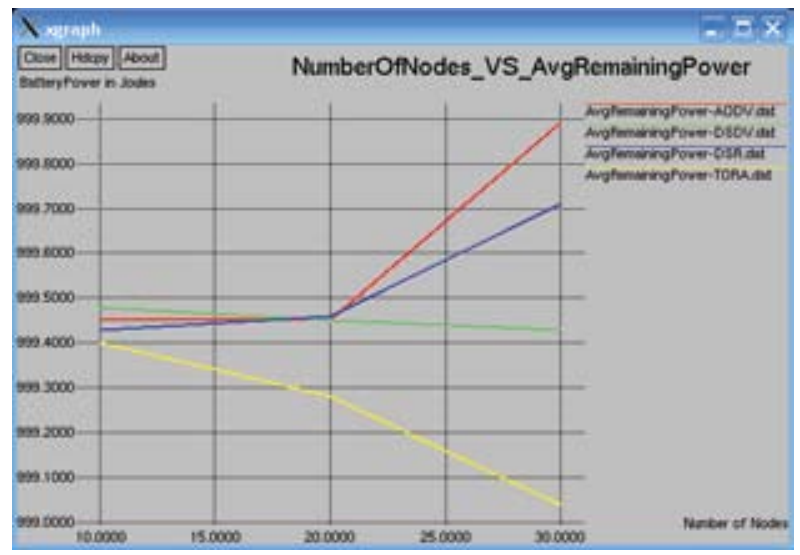

Figure 3. The average remaining power versus the number of nodes using 30 nodes.

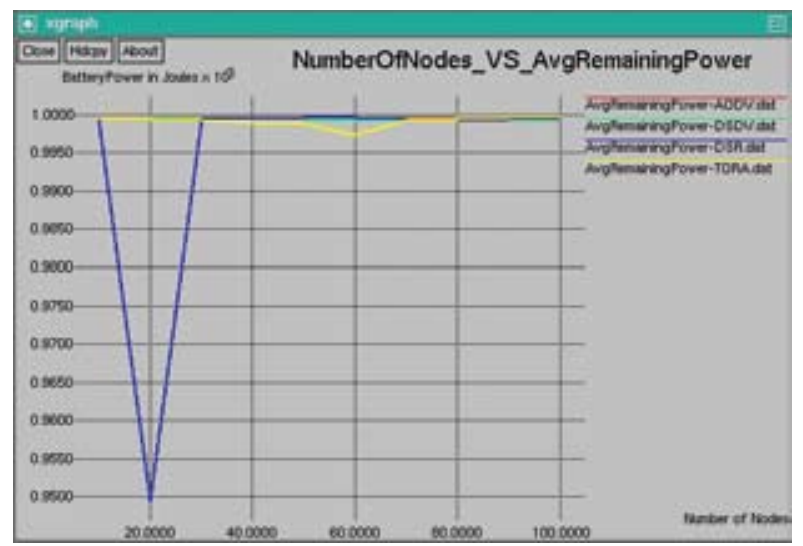

Figure 4. The average remaining power versus the number of nodes using 100 nodes.

Figure 3 shows that the energy efficiency of networks using AODV and DSR increases significantly when the number of nodes exceeds 20 . On the contrary, the energy efficiency of a network using the TORA protocol drops sharply whilst that of DSDV based network shows a gentle decrease with increasing number of nodes.

Figure 4 shows exactly the same results with the graph of

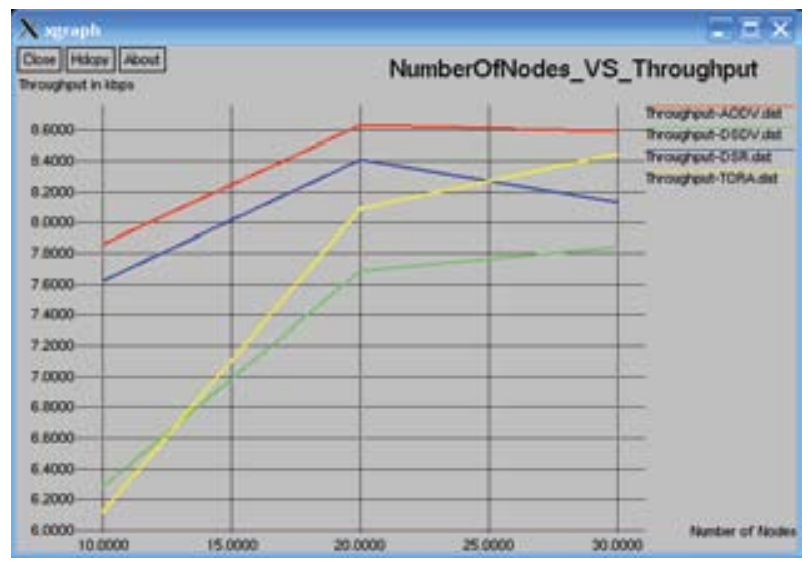

Figure 5. The number of nodes versus throughput using 30 nodes.

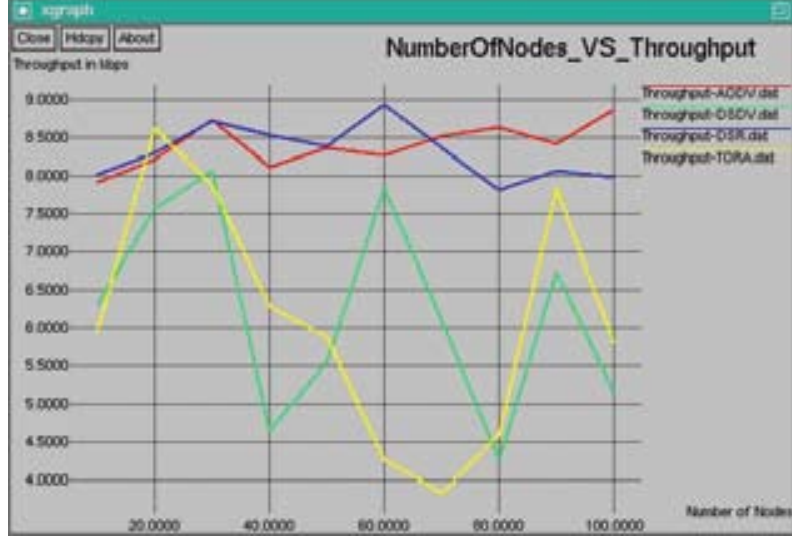

Figure 6. The number of nodes versus throughput using 100 nodes.

figure 2. Figure 4 depicts that the remaining power when DSR protocol is decreases significantly when the number of nodes is 20, whilst the other three protocols have the same behaviour.

Figure 5 shows the throughput of AODV protocol becoming stable when the number of nodes exceeds 20 while the DSR decreases significally. On the other hand the throughput of TORA and DSDV increases rapidly when the nodes exceeds 20 .

Figure 6 shows that the throughput of AODV and DSR protocols have almost the same behaviour. On the other hand TORA and DSDV presents the same behaviour when the number of nodes are 20 and 40 but after that, the thrughput of TORA decreasing rapidly when the umber of nodes are 60 and 70 and increasing rapidly when the number of nodes is near to 100 .

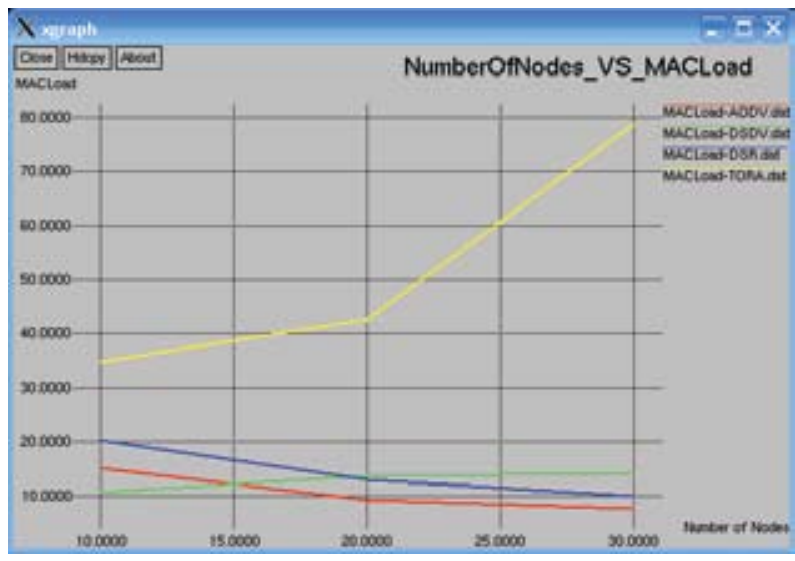

Figure 7. The MAC Load using 30 nodes.

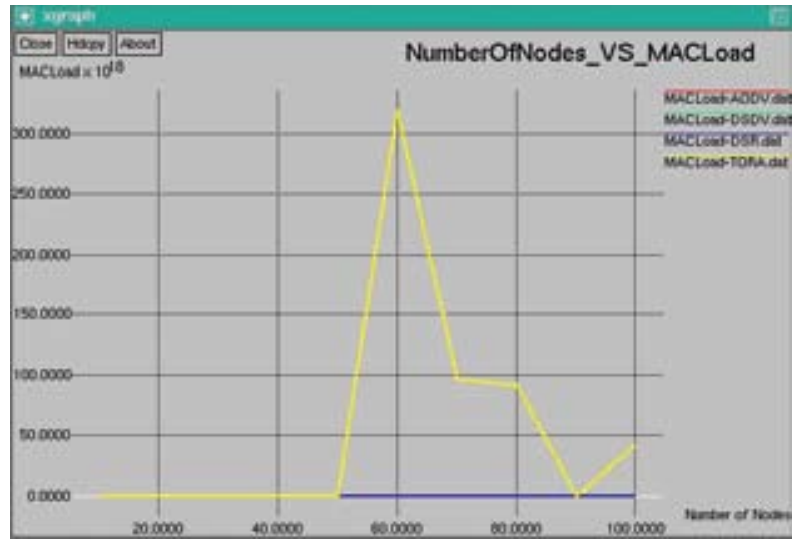

Figure 8. The MAC Load using 100 nodes. 
Figure 7 shows that the MAC Load increases rapidly when the number of nodes exceeds 20 for TORA protocol. For the other three protocols the MAC Load is stable and stay in low level.

Figure 8 shows that the MAC Load icrease rapidly when the number of nodes exceeds 50 for TORA protocol and become stable when the number of nodes is bigger than 90 . For the other three protocols the MAC Load is stable in all kind of networks.

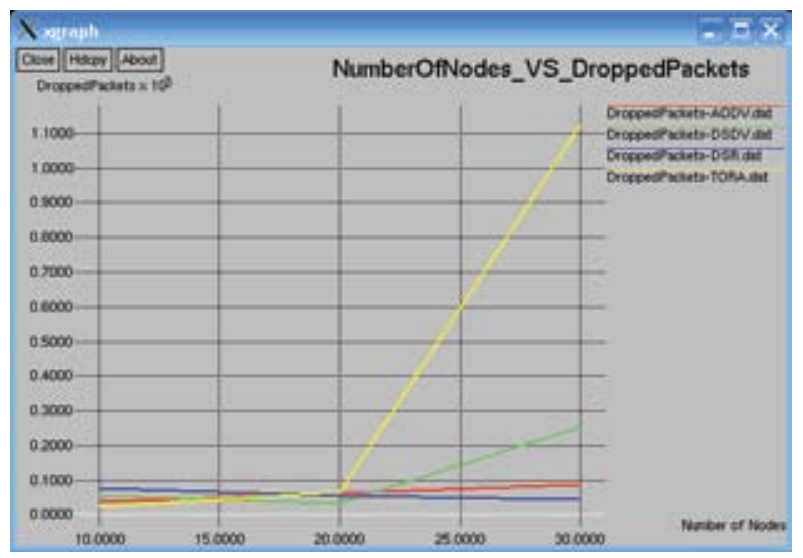

Figure 9. The Dropped Packets using 30 nodes.

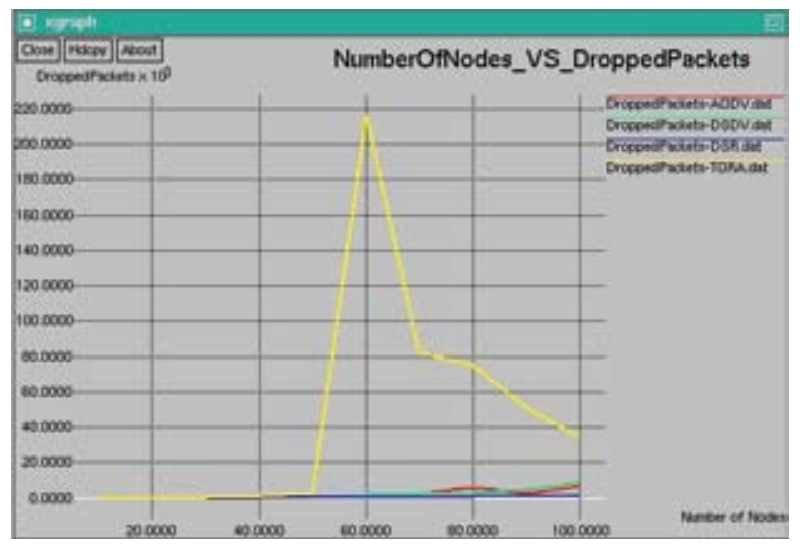

Figure 10. The Dropped Packets using 100 nodes.

Figures 9 and 11 show that when the number of nodes exceeds 20 the network performance deteriorates significantly resulting in maximum loss of information (Dropped Packets \& Dropped Bytes). On the contrary, the AODV and DSR protocols have the minimum loss of information of all the 4 protocols.

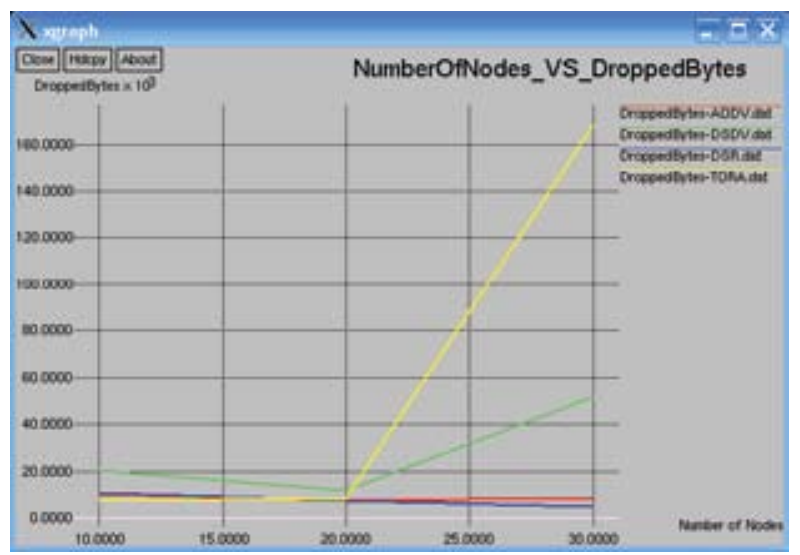

Figure 11. The Dropped Bytes using 30 nodes.

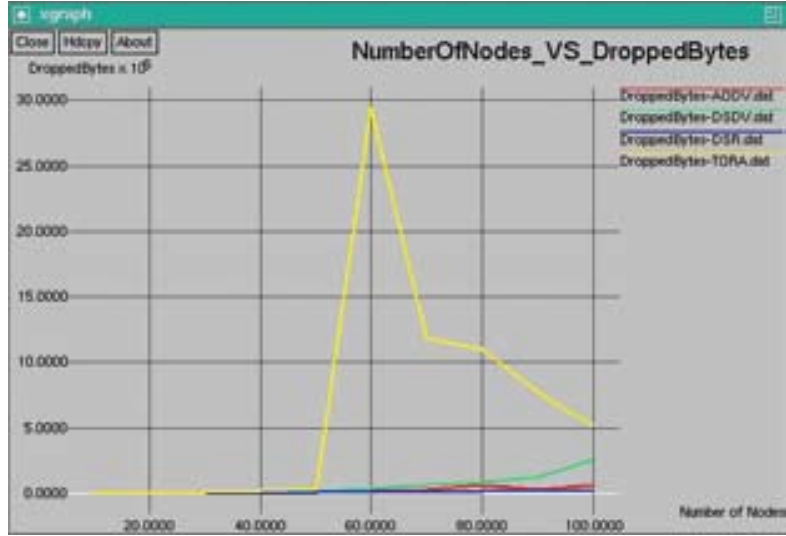

Figure 12. The Dropped Bytes using 100 nodes.

Figure 10 and 12 show that the TORA protocol becomes inefficient when the network consists of more than 20 nodes for low density network while for high density network becomes ineffcient when the network consists more than 50 nodes.

In an attempt to obtain meaningful results during this study using the TORA protocol in ns2 [9][10], it was necessary to introduce a small patch/modification. One obvious conclusion that can be drawn from this study is that the ns2 implementation of TORA still seems to be flawed and in need of further development. For instance, it worked only with 4 or 5 nodes in some ideal node configuration.

As per the simulations made on ns, it can be concluded that TORA, AODV and DSDV perform well in small scale networks. Thus, it may be considered for further enhancement in terms of seeking to improve power consumption.

\section{Conclusion and Future Work}

This study has evaluated four ad-hoc routing protocols in different network environment taking into consideration node mobility. Overall, the findings show that the energy consumption and throughput in small size networks did not reveal any significant differences. However, for medium and large ad-hoc networks the TORA performance proved to be inefficient in this study. In particular, the performance of AODV, DSDV and DSR in small size networks was comparable. But in medium and large size networks, the AODV and DSR produced good results and the performance of AODV in terms of throughput is good in all the scenarios that have been investigated.

Whilst experimenting with TORA in ns2, TORA seems to be providing poor performance which is very controversial to the theoretical expectations. Finding the reason for this poor behaviours' and correcting it is not simple. It is more than complex that of writing a new protocol. 


\section{References}

1. S. Giannoulis, C. Antonopoulos, E. Topalis, S. Koubias, ZRP versus DSR and TORA: A comprehensive survey on ZRP performance, IEEE Transactions on Industrial Informatics Vol. 3, No. 1, pp. 63-72 (Feb. 2007).

2. D. Maltz, Y. Hu, The Dynamic Source Routing Protocol for Mobile Ad Hoc Networks, Internet Draft, Available: http://www.ietf.org/internetdrafts/draft-ietf-manet-dsr-10.txt, July 2004.

3. C. Perkins and E. Royer, Ad Hoc On-demand Distance Vector (AODV) Routing, Internet Draft, MANET working group, draft-ietf-manetaodv-05.txt, March 2000.

4. A. H. Abd Rahman, Z. A. Zukarnain, Performance Comparison of AODV, DSDV and I-DSDV Routing Protocols in Mobile Ad Hoc Networks, European Journal of Scientific Research Vol. 31, No. 4, pp. 556-576 (June 2009).
5. L. Junhai, X. Liu, Y. Danxia, Research on multicast routing protocols for mobile ad-hoc networks, Science Direct Vol. 52 Issue 5, pp. 988-997 (April 2008).

6. M. Abolhasan, T. Wysocki, E. Dutkiewicz, A review of routing protocols for mobile ad hoc networks, Science Direct Vol. 2, Issue 1, pp. 1-22 (January 2004).

7. Ns-2 network simulator, http://www.isi.edu/nsnam/ns/, 1998,

8. A. Rahman, S. Islam, A. Talevski, Performance Measurement of various Routing Protocol in Ad-Hoc Network, IMECS, Vol. 1, pp. 321-323 (March 2009).

9. CMU Monarch extensions to ns-2, http://www.monarch.cs.cmu.edu/cmuns.html, 1999.

10. Marc Greis' Tutorial for the UCB/LBNL/VINT Network Simulator "ns". 